\title{
Diabetes mellitus and tuberculosis—a double whammy
}

\author{
S. V. Madhu ${ }^{1}$
}

Published online: 27 May 2017

(C) Research Society for Study of Diabetes in India 2017

India has the second highest prevalence of diabetes mellitus in the world with over 69.2 million diabetes subjects [1]. So also, India accounts for a significant proportion of cases of tuberculosis in the world. It is also estimated that the highest number of cases of tuberculosis associated with diabetes are reported from India and a large proportion of diabetic patients in our country suffer from tuberculosis [2-4]. This suggests a huge double burden of diabetes and tuberculosis in our country.

The study by Hussain et al. from Odisha in this issue is from Eastern India and reports that $14 \%$ of active tuberculosis patients in the region have diabetes mellitus and a further $24 \%$ have prediabetes. The authors also report that nearly half of those detected with diabetes and over half of those detected with prediabetes were unaware of their glucose intolerance. The study thus underlines the need for and demonstrates the feasibility of screening for early diagnosis and treatment of diabetes mellitus if we have to improve tuberculosis-related outcomes.

Prevalence of diabetes in patients with tuberculosis from different states of India has varied from $11.6 \%$ in Punjab to $44 \%$ in Kerala [5, 6]. Southern Indian states (Kerala 44\%, Tamil Nadu 25\%, and Pudicherry 29\%) have reported higher prevalence of diabetes mellitus in such cases as compared to the Western (Ahmadabad 15\%) or North Indian (Punjab $11.6 \%)$ states [5-8].

S. V. Madhu

drsvmadhu@gmail.com

1 Department of Endocrinology, Centre for Diabetes, Endocrinology and Metabolism, University College of Medical Sciences - GTB Hospital, Delhi, India
The regional variation in the prevalence of coexistent diabetes and tuberculosis within India is both striking and interesting. Whether this merely reflects the regional differences in prevalence of diabetes within the country is unclear. Data from INDIAB [9] indicates that the prevalence of diabetes mellitus is lower in Maharashtra in the West and in rural Jharkhand in the East compared to that in South and North India. However, this does not fully explain the regional variation of cases of diabetes and tuberculosis in the country, and it will be interesting to investigate if other regional factors favoring the coexistence of diabetes mellitus and tuberculosis have a role to play.

The coexistence of diabetes mellitus and tuberculosis has adverse consequences for both conditions. Diabetes predisposes to tuberculosis due to the profound effects of hyperglycemia on body defense mechanisms including suppression of cell-mediated immune mechanisms [10]. It is also well known that presence of diabetes mellitus also alters the way pulmonary tuberculosis manifests and adversely affects clinical and treatment outcomes [11]. It also results in slow conversion to a sputum-negative state [12]. Similarly, the presence of active tuberculosis leads to unmasking of higher levels of glucose intolerance and poses serious challenges to glucose control among diabetic patients. Tuberculosis is an important cause of insulinization and hospitalization among diabetic patients that also increases the cost of diabetes care besides affecting quality of life in these patients.

The original article by Dwivedi et al. in this issue evaluates the CT-based radiological manifestations of pulmonary tuberculosis among diabetic patients. The study confirms higher prevalence of lower lobe consolidation and cavitary lesions in them when compared to those without diabetes. The patients of tuberculosis with diabetes mellitus also showed higher rates of endobronchial spread (57\%) compare to nondiabetic subjects $(30 \%)$. The authors conclude that the 
presence of lower lobe consolidation or cavitation in a case of pulmonary tuberculosis should alert the clinician to coexistent diabetes mellitus.

While the association of diabetes mellitus and tuberculosis poses a serious challenge to our efforts to control both diseases, the coexistence of these two conditions also provides a huge public health opportunity. Strategies for early detection of diabetes and tuberculosis when they coexist with each other will be critical in controlling the rising prevalence and impact of both. National guidelines for bi-directional screening of diabetes mellitus and tuberculosis when patients present with either of these two conditions is a welcome step in this direction.

There is also a need to undertake further studies particularly cohort studies to unravel some of the missing links in the coexistence of diabetes mellitus and tuberculosis. More importantly, there is an urgent need to develop clinical practice recommendations for diagnosis, prevention, and control of diabetes mellitus-tuberculosis to facilitate a uniform and effective approach that will help contain this twin problem.

\section{References}

1. IDF diabetes atlas. 7th Edition. Brussels: International Diabetes Federation; 2015.

2. Harries AD, Satyanarayana S, Kumar AM, et al. Epidemiology and interaction of diabetes mellitus and tuberculosis and challenges for care: a review. Public Health Action. 2013;3(Suppl 1):S3-9.

3. Lonnroth K, Roglic G, Harries AD. Improving tuberculosis prevention and care through addressing the global diabetes epidemic: from evidence to policy and practice. Lancet Diabetes Endocrinol. 2014;2(9):730-9.
4. Zheng $\mathrm{C}, \mathrm{Hu} \mathrm{M}$, Gao F. Diabetes and pulmonary tuberculosis: a global overview with special focus on the situation in Asian countries with high TB-DM burden. Glob Health Action. 2017;10(1):111.

5. Singh SP, Singh SP, Kishan J, Kaur S, Ramana S. Association of tuberculosis and diabetes mellitus: an analysis of 1000 consecutively admitted cases in a tertiary care hospital of North India. Pan Afr Med J. 2016;24:4.

6. Balakrishnan S, Vijayan S, Nair S, et al. High diabetes prevalence among tuberculosis cases in Kerala, India. PLoS ONE. 2012;7(10): e46502.

7. Viswanathan V, Kumpatla S, Aravindalochanan V, et al. Prevalence of diabetes and pre-diabetes and associated risk factors among tuberculosis patients in India. PLoS One. 2012;7(7):e41367.

8. Raghuraman S, Vasudevan KP, Govindarajan S, Chinnakali P, Panigrahi KC. Prevalence of diabetes mellitus among tuberculosis patients in urban Puducherry. N Am J Med Sci. 2014;Vol 6:30-4.

9. Anjana RM, Pradeepa R, Deepa M, Datta M, Sudha V, Unnikrishnan R, Bhansali A, Joshi SR, Joshi PP, Yajnik CS, Dhandhania VK, Nath LM, Das AK, Rao PV, Madhu SV, Shukla DK, Kaur T, Priya M, Nirmal E, Parvathi SJ, Subhashini S, Subashini R, Ali MK, Mohan V, ICMR-INDIAB Collaborative Study Group. Prevalence of diabetes and prediabetes (impaired fasting glucose and/or impaired glucose tolerance) in urban and rural India: phase I results of the Indian Council of Medical Research-INdia DIABetes (ICMR-INDIAB) study. Diabetologia. 2011;54:3022-7.

10. Hodgson K, Morris J, Bridson T, Govan B, Rush C, Ketheesan N. Immunological mechanisms contributing to the double burden of diabetes and intracellular bacterial infections. Immunology. 2015;144(2):171-85.

11. Perez-Navarro LM, Restrepo BI, Fuentes-Dominguez FJ, et al. The effect size of type 2 diabetes mellitus on tuberculosis drug resistance and adverse treatment outcomes. Tuberculosis (Edinb). 2017;103: 83-91.

12. Restrepo BI, Fisher-Hoch SP, Smith B, et al. Mycobacterial clearance from sputum is delayed during the first phase of treatment in patients with diabetes. AmJTrop Med Hyg. 2008;79(4):541-4. 